\title{
On two - parameter lindley distribution and its applications to model lifetime data
}

\begin{abstract}
In this paper some of the important mathematical properties including moment generating function, mean deviations, order statistics, Bonferroni and Lorenz curves, Renyi entropy and stress strength reliability of two-parameter Lindley distribution (TPLD) of Shanker \& Mishra ${ }^{1}$ have been discussed. Its goodness of fit over exponential and Lindley distributions have been illustrated with some real lifetime data-sets and found that TPLD is preferable over exponential and Lindley distributions for modeling lifetime data-sets.
\end{abstract}

Keywords: mean deviations; order statistics, bonferroni and lorenz curves, entropy, stress-strength reliability, goodness of fit
Volume 3 Issue I - 2016

\author{
Rama Shanker,' Hagos Fesshaye, ${ }^{2}$ Shambhu \\ Sharma ${ }^{3}$ \\ 'Department of Statistics, Eritrea Institute of Technology, Eritrea \\ ${ }^{2}$ Department of Economics, College of Business and Economics, \\ Eritrea \\ ${ }^{3}$ Department of Mathematics, Dayalbagh Educational Institute, \\ India
}

Correspondence: Rama Shanker, Eritrea Institute of Technology, Asmara, Eritrea, Email shankerrama2009@gmail.com

Received: October 28, 2015 | Published: January 02, 2016

\section{Introduction}

The probability density function (p.d.f.) and the cumulative distribution function (c.d.f.) of Lindley ${ }^{2}$ distribution, introduced in the context of Bayesian analysis as a counter example of fiducial statistics, are given by

$$
\begin{gathered}
f(x ; \theta)=\frac{\theta^{2}}{\theta+1}(1+x) e^{-\theta x} ; x>0, \theta>0 \\
F(x ; \theta)=1-\left[1+\frac{\theta x}{\theta+1}\right] e^{-\theta x} ; x>0, \theta>0
\end{gathered}
$$

The detailed study about its mathematical properties, estimation of parameter and application showing the superiority of Lindley distribution over exponential distribution for the waiting times before service of the bank customers has been done by Ghitany et al. ${ }^{3}$. The Lindley distribution has been generalized extended and modified by different researchers including ${ }^{1,4-20}$ are some among others.

The probability density function (p.d.f.) and cumulative distribution function (c.d.f) of two-parameter Lindley distribution (TPLD) of Shanker \& Mishra $^{1}$ are given by

$$
\begin{gathered}
f(x ; \alpha, \theta)=\frac{\theta^{2}}{\alpha \theta+1}(\alpha+x) e^{-\theta x} ; x>0, \theta>0, \alpha \theta>-1 \\
F(x ; \alpha, \theta)=1-\left[\frac{1+\alpha \theta+\theta x}{\alpha \theta+1}\right] e^{-\theta x} ; x>0, \theta>0, \alpha \theta>-1
\end{gathered}
$$

At $\alpha=1$, both (1.3) and (1.4) reduce to the corresponding expressions (1.1) and (1.2) of Lindley distribution. The first two moments about origin and the variance of TPLD of Shanker \& Mishra $^{1}$ are given by

$$
\mu_{1}^{\prime}=\frac{\alpha \theta+2}{\theta(\alpha \theta+1)}
$$

$$
\begin{aligned}
& \mu_{2}^{\prime}=\frac{2(\alpha \theta+3)}{\theta^{2}(\alpha \theta+1)} \\
& \mu_{2}=\frac{\alpha^{2} \theta^{2}+4 \alpha \theta+2}{\theta^{2}(\alpha \theta+1)^{2}}
\end{aligned}
$$

At $\alpha=1$, these moments reduce to the corresponding moments of Lindley distribution. Shanker \& Mishra ${ }^{1}$ have derived and discussed some of its mathematical properties such as shape, moments, coefficient of variation, coefficient of skewness and kurtosis, hazard rate function, mean residual life function and stochastic orderings. They have also discussed the estimation of its parameters using maximum likelihood estimation and method of moments and its goodness of fit over Lindley distribution. It has been observed that many important mathematical properties of this distribution have not been studied.

In the present paper some of the important mathematical properties including moment generating function, mean deviations, order statistics, Bonferroni and Lorenz curves, Renyi entropy and stress strength reliability of TPLD of Shanker \& Mishra ${ }^{1}$ have been derived and discussed. Its goodness of fit over exponential and Lindley distributions have been illustrated with some real lifetime data-sets and found that TPLD gives better fit than exponential and Lindley distributions.

\section{Moment generating function}

The moment generating function, $\left(M_{X}(t)\right)$ of TPLD (1.3) can be obtained as

$$
\begin{gathered}
M_{X}(t)=\frac{\theta^{2}}{\alpha \theta+1} \int_{0}^{\infty} e^{-(\theta-t)}(\alpha+x) d x \\
=\frac{\theta^{2}}{\alpha \theta+1}\left[\frac{\alpha}{\theta-t}+\frac{1}{(\theta-t)^{2}}\right]
\end{gathered}
$$




$$
\begin{gathered}
=\frac{\theta^{2}}{\alpha \theta+1}\left[\frac{\alpha}{\theta} \sum_{k=0}^{\infty}\left(\frac{t}{\theta}\right)^{k}+\frac{1}{\theta^{2}} \sum_{k=0}^{\infty}\left(\begin{array}{c}
k+1 \\
k
\end{array}\right)\left(\frac{t}{\theta}\right)^{k}\right] \\
=\sum_{k=0}^{\infty}\left(\frac{\alpha \theta+1+k}{\alpha \theta+1}\right)\left(\frac{t}{\theta}\right)^{k}
\end{gathered}
$$

It can be easily seen that the expression for $\mu_{r}$ ' obtained as the coefficient of $\frac{t^{r}}{r !} \operatorname{in} M_{X}(t)$ is given as

$$
\mu_{r}^{\prime}=\frac{r !(\alpha \theta+r+1)}{\theta^{r}(\alpha \theta+1)} ; r=1,2,3, \ldots
$$

For $\alpha=1, \quad \mu_{r}^{\prime}$ reduces to the corresponding $\mu_{r}^{\prime}$ of Lindley distribution.

\section{Mean deviations}

The amount of scatter in a population is measured to some extent by the totality of deviations usually from mean and median. These are known as the mean deviation about the mean and the mean deviation about the median defined by $\delta_{1}(x)=\int_{0}^{\infty}|x-\mu| f(x) d x$ and $\delta_{2}(x)=\int_{0}^{\infty}|x-M| f(x) d x$, respectively, where $\mu=E(X)$ and $M=\operatorname{Median}(X)$.

The measures $\delta_{1}(X)$ and $\delta_{2}(X)$ can be calculated using the relationships

$$
\begin{aligned}
& \delta_{1}(X)=\int_{0}^{\mu}(\mu-x) f(x) d x+\int_{\mu}^{\infty}(x-\mu) f(x) d x \\
& =\mu F(\mu)-\int_{0}^{\mu} x f(x) d x-\mu[1-F(\mu)]+\int_{\mu}^{\infty} x f(x) d x \\
& =2 \mu F(\mu)-2 \mu+2 \int_{\mu}^{\infty} x f(x) d x \\
& \text { and } \begin{aligned}
\delta_{2}(X) & =\int_{0}^{M}(M-x) f(x) d x+\int_{M}^{\infty}(x-M) f(x) d x \\
=M F(M)-\int_{0}^{M} x f(x) d x & \\
= & =-\mu+2 \int_{M}^{\infty} x f(x) d x \\
& =\mu-2 \int_{0}^{M} x f(x) d x
\end{aligned}
\end{aligned}
$$

Using p.d.f. (1.3), and expression for mean of two-parameter Lindley distribution, we have

$$
\int_{0}^{\mu} x f(x) d x=\mu-\frac{\left\{\theta^{2}\left(\mu^{2}+\alpha \mu\right)+2 \theta \mu+(\alpha \theta+2)\right\} e^{-\theta \mu}}{\theta(\alpha \theta+1)}
$$

$$
\int_{0}^{M} x f(x) d x=\mu-\frac{\left\{\theta^{2}\left(M^{2}+\alpha M\right)+2 \theta M+(\alpha \theta+2)\right\} e^{-\theta M}}{\theta(\alpha \theta+1)}
$$

Using expressions from (3.1), (3.2), (3.3) and (3.4) and little algebraic simplification, the mean deviation about mean, $\delta_{1}(X)$ and the mean deviation about median, $\delta_{2}(X)$ of TPLD (1.3) are obtained as

$$
\delta_{1}(X)=\frac{2(\theta \mu+\alpha \theta+2) e^{-\theta \mu}}{\theta(\alpha \theta+1)}
$$

and

$$
\delta_{2}(X)=\frac{2\left\{\theta^{2}\left(M^{2}+\alpha M\right)+2 \theta M+(\alpha \theta+2)\right\} e^{-\theta M}}{\theta(\alpha \theta+1)}-\mu
$$

It can be easily seen that expressions (3.5) and (3.6) of TPLD (1.3) reduce to the corresponding expressions of Lindley distribution at $\alpha=1$.

\section{Order statistics}

Let $X_{1}, X_{2}, \ldots, X_{n}$ be a random sample of size $n$ from twoparameter Lindley distribution (1.3). Let $X_{(1)}<X_{(2)}<\ldots<X_{(n)}$ denote the corresponding order statistics. The p.d.f. and the c.d.f. of the $k$ th order statistic, say $Y=X_{(k)}$ are given by

$$
\begin{aligned}
& f_{Y}(y)=\frac{n !}{(k-1) !(n-k) !} F^{k-1}(y)\{1-F(y)\}^{n-k} f(y) \\
& =\frac{n !}{(k-1) !(n-k) !} \sum_{l=0}^{n-k}\left(\begin{array}{c}
n-k \\
l
\end{array}\right)(-1)^{l} F^{k+l-1}(y) f(y) \\
& \text { and } \\
& \qquad F_{Y}(y)=\sum_{j=k}^{n}\left(\begin{array}{c}
n \\
j
\end{array}\right) F^{j}(y)\{1-F(y)\}^{n-j} \\
& =\sum_{j=k}^{n} \sum_{l=0}^{n-j}\left(\begin{array}{c}
n \\
j
\end{array}\right)\left(\begin{array}{c}
n-j \\
l
\end{array}\right)(-1)^{l} F^{j+l}(y),
\end{aligned}
$$

respectively, for $k=1,2,3, \ldots, n$.

Thus, the p.d.f. and the c.d.f of the $k$ th order statistics of TPLD (1.3) are obtained as

$$
\begin{aligned}
& f_{Y}(y)=\frac{n ! \theta^{2}(\alpha+x) e^{-\theta x}}{(\alpha \theta+1)(k-1) !(n-k) !} \sum_{l=0}^{n-k}\left(\begin{array}{c}
n-k \\
l
\end{array}\right)(-1)^{l} \times\left[1-\frac{1+\alpha \theta+\theta x}{\alpha \theta+1} e^{-\theta x}\right]^{k+l-1} \\
& \text { and } \\
& F_{Y}(y)=\sum_{j=k}^{n} \sum_{l=0}^{n-j}\left(\begin{array}{c}
n \\
j
\end{array}\right)\left(\begin{array}{c}
n-j \\
l
\end{array}\right)(-1)^{l}\left[1-\frac{1+\alpha \theta+\theta x}{\alpha \theta+1} e^{-\theta x}\right]^{j+l}
\end{aligned}
$$

It can be easily verified that the expressions for the p.d.f. and c.d.f. of the $k$ th order statistics of TPLD (1.3) reduce to the expressions for the p.d.f. and c.d.f. of the $k$ th order statistics of Lindley distribution at $\alpha=1$ 


\section{Bonferroni and lorenz curves}

The Bonferroni and Lorenz curves ${ }^{21}$ and Bonferroni and Gini indices have applications not only in economics to study income and poverty, but also in other fields like reliability, demography, insurance and medicine. The Bonferroni and Lorenz curves are defined as

$$
\begin{aligned}
& B(p)=\frac{1}{p \mu} \int_{0}^{q} x f(x) d x=\frac{1}{p \mu}\left[\int_{0}^{\infty} x f(x) d x-\int_{q}^{\infty} x f(x) d x\right]=\frac{1}{p \mu}\left[\mu-\int_{q}^{\infty} x f(x) d x\right] \\
& \text { and } \\
& L(p)=\frac{1}{\mu} \int_{0}^{q} x f(x) d x=\frac{1}{\mu}\left[\int_{0}^{\infty} x f(x) d x-\int_{q}^{\infty} x f(x) d x\right]=\frac{1}{\mu}\left[\mu-\int_{q}^{\infty} x f(x) d x\right]
\end{aligned}
$$

respectively or equivalently

$$
B(p)=\frac{1}{p \mu} \int_{0}^{p} F^{-1}(x) d x
$$

and

$$
L(p)=\frac{1}{\mu} \int_{0}^{p} F^{-1}(x) d x
$$

respectively, where $\mu=E(X)$ and $q=F^{-1}(p)$.

The Bonferroni and Gini indices are thus defined as

$$
\begin{aligned}
B & =1-\int_{0}^{1} B(p) d p \\
\text { and } G & =1-2 \int_{0}^{1} L(p) d p
\end{aligned}
$$

respectively.

Using p.d.f. (1.3), we get

$$
\int_{q}^{\infty} x f(x) d x=\frac{\left\{\theta^{2}\left(q^{2}+\alpha q\right)+2 \theta q+(\alpha \theta+2)\right\} e^{-\theta q}}{\theta(\alpha \theta+1)}
$$

Now using equation (5.7) in (5.1) and (5.2), we get

$$
\begin{aligned}
& B(p)=\frac{1}{p}\left[1-\frac{\left\{\theta^{2}\left(q^{2}+\alpha q\right)+2 \theta q+(\alpha \theta+2)\right\} e^{-\theta q}}{\alpha \theta+2}\right] \\
& \text { and } L(p)=1-\frac{\left\{\theta^{2}\left(q^{2}+\alpha q\right)+2 \theta q+(\alpha \theta+2)\right\} e^{-\theta q}}{\alpha \theta+2}
\end{aligned}
$$

Now using equations (5.8) and (5.9) in (5.5) and (5.6), the Bonferroni and Gini indices of TPLD (1.3) are obtained as

$$
\begin{aligned}
& B=1-\frac{\left\{\theta^{2}\left(q^{2}+\alpha q\right)+2 \theta q+(\alpha \theta+2)\right\} e^{-\theta q}}{\alpha \theta+2} \\
& G=-1+\frac{2\left\{\theta^{2}\left(q^{2}+\alpha q\right)+2 \theta q+(\alpha \theta+2)\right\} e^{-\theta q}}{\alpha \theta+2}
\end{aligned}
$$

The Bonferroni and Gini indices of Lindley distribution are particular cases of the Bonferroni and Gini indices (5.10) and (5.11) of TPLD (1.3) for $\alpha=1$.

\section{Renyi entropy}

An entropy of a random variable $X$ is a measure of variation of uncertainty. A popular entropy measure is Renyi entropy. ${ }^{22}$ If $X$ is a continuous random variable having probability density function , then Renyi entropy is defined as

$$
T_{R}(\gamma)=\frac{1}{1-\gamma} \log \left\{\int f^{\gamma}(x) d x\right\}
$$

where $\gamma>0$ and $\gamma \neq 1$.

Thus, the Renyi entropy for TPLD (1.3) can be obtained as

$$
\begin{aligned}
& T_{R}(\gamma)=\frac{1}{1-\gamma} \log \left[\int_{0}^{\infty} \frac{\theta^{2 \gamma}}{(\alpha \theta+1)^{\gamma}}(\alpha+x)^{\gamma} e^{-\theta \gamma x} d x\right] \\
& =\frac{1}{1-\gamma} \log \left[\int_{0}^{\infty} \frac{\alpha^{\gamma} \theta^{2 \gamma}}{(\alpha \theta+1)^{\gamma}}\left(1+\frac{x}{\alpha}\right)^{\gamma} e^{-\theta \gamma x} d x\right] \\
& =\frac{1}{1-\gamma} \log \left[\int_{0}^{\infty}\left(\frac{\alpha}{\alpha \theta+1}\right)^{\gamma} \theta^{2 \gamma} \sum_{j=0}^{\infty}\left(\begin{array}{l}
\gamma \\
j
\end{array}\right)\left(\frac{x}{\alpha}\right)^{j} e^{-\theta \gamma x} d x\right] \\
& =\frac{1}{1-\gamma} \log \left[\sum_{j=0}^{\infty}\left(\begin{array}{l}
\gamma \\
j
\end{array}\right)\left(\frac{\alpha}{\alpha \theta+1}\right)^{\gamma} \frac{\theta^{2 \gamma}}{\alpha^{j}} \int_{0}^{\infty} e^{-\theta \gamma x} x^{j+1-1} d x\right] \\
& =\frac{1}{1-\gamma} \log \left[\sum_{j=0}^{\infty}\left(\begin{array}{l}
\gamma \\
j
\end{array}\right) \frac{\alpha^{\gamma-j} \theta^{2 \gamma}}{(\alpha \theta+1)^{\gamma}} \frac{\Gamma(j+1)}{(\theta \gamma)^{j+1}}\right] \\
& =\frac{1}{1-\gamma} \log \left[\sum_{j=0}^{\infty}\left(\begin{array}{l}
\gamma \\
j
\end{array}\right) \frac{\alpha^{\gamma-j} \theta^{2 \gamma-j-1}}{(\alpha \theta+1)^{\gamma}} \frac{\Gamma(j+1)}{(\gamma)^{j+1}}\right]
\end{aligned}
$$

The Renyi entropy of Lindley distribution is a particular case of the Renyi entropy TPLD at $\alpha=1$.

\section{Stress-strength reliability}

The stress- strength reliability describes the life of a component which has random strength $X$ that is subjected to a random stress $Y$. When the stress applied to it exceeds the strength, the component fails instantly and the component will function satisfactorily till $X>Y$ . Therefore, $R=P(Y<X)$ is a measure of component reliability and in statistical literature it is known as stress-strength parameter. It has wide applications in almost all areas of knowledge especially in engineering such as structures, deterioration of rocket motors, static fatigue of ceramic components, aging of concrete pressure vessels etc.

Let $X$ and $Y$ be independent strength and stress random variables having TPLD (1.3) with parameter $\left(\alpha_{1}, \theta_{1}\right)$ and $\left(\alpha_{2}, \theta_{2}\right)$ 
respectively. Then the stress-strength reliability $R$ is obtained as

$$
\begin{gathered}
R=P(Y<X)=\int_{0}^{\infty} P(Y<X \mid X=x) f_{X}(x) d x \\
=\int_{0}^{\infty} f\left(x ; \alpha_{1}, \theta_{1}\right) F\left(x ; \alpha_{2}, \theta_{2}\right) d x \\
=1-\frac{\theta_{1}^{2}\left[2 \theta_{2}+\left(\alpha_{1} \theta_{2}+\alpha_{2} \theta_{2}+1\right)\left(\theta_{1}+\theta_{2}\right)+\alpha_{1}\left(\alpha_{2} \theta_{2}+1\right)\left(\theta_{1}+\theta_{2}\right)^{2}\right]}{\left(\alpha_{1} \theta_{1}+1\right)\left(\alpha_{2} \theta_{2}+1\right)\left(\theta_{1}+\theta_{2}\right)^{2}} .
\end{gathered}
$$

The expression of stress-strength reliability of Lindley distribution is a particular case of the expression of stress-strength reliability of $\operatorname{TPLD}(1.3)$ at $\alpha_{1}=\alpha_{2}=1$.

\section{Estimation of parameters}

\section{a. Method of moment estimate of parameters}

The TPLD (1.3) has two parameters to be estimated and so the first two moments about origin are required to estimate parameters. Using the first two moments about origin, we have

$$
\frac{\mu_{2}^{\prime}}{\left(\mu_{1}^{\prime}\right)^{2}}=k(\text { Say })=\frac{2(\alpha \theta+3)(\alpha \theta+1)}{(\alpha \theta+2)^{2}}
$$

Taking $b=\alpha \theta$, we get

$$
\frac{\mu_{2}^{\prime}}{\left(\mu_{1}^{\prime}\right)^{2}}=\frac{2(b+3)(b+1)}{(b+2)^{2}}=\frac{2 b^{2}+8 b+6}{b^{2}+4 b+4}=k
$$

This gives a quadratic equation in as

$$
(2-k) b^{2}+4(2-k) b+2(3-2 k)=0
$$

Replacing the first and second moments about origin $\mu_{1}^{\prime}$ and $\mu_{2}{ }^{\prime}$ by their respective sample moments, an estimate of $k$ can be obtained and substituting the value of $k$ in equation (8.1.2), an estimate of can be obtained. Substituting this estimate of in the expression for the mean of TPLD (1.3), moment estimate $\tilde{\theta}$ of $\theta$ can be obtained as

$$
\tilde{\theta}=\left(\frac{b+2}{b+1}\right) \frac{1}{\bar{x}}
$$

Finally, moment estimate $\tilde{\alpha}$ of $\alpha$ can be obtained as

$$
\tilde{\alpha}=\frac{b}{\tilde{\theta}}
$$

\section{b. Maximum likelihood estimate of parameters}

Let $\left(x_{1}, x_{2}, x_{3}, \ldots, x_{n}\right)$ be a random sample from TPLD (1.3) Let $f_{x}$ be the observed frequency in the sample corresponding to $X=x(x=1,2,3, \ldots k)$ such that $\sum_{x=1}^{k} f_{x}=n$, where $k$ is the largest observed value having non-zero frequency. The likelihood function, $L$ of TPLD (1.3) is given by

$$
L=\left(\frac{\theta^{2}}{\alpha \theta+1}\right)^{n} \prod_{i=1}^{n}(\alpha+x)^{f_{x}} e^{-n \theta \bar{x}}
$$

The log likelihood function is thus obtained as

$\log L=n \log \theta^{2}-n \log (\alpha \theta+1)+\sum_{x=1}^{k} f_{x} \log (\alpha+x)-n \theta \bar{x}$

where $\bar{x}$ is the sample mean.

The two log likelihood equations are obtained as

$$
\begin{gathered}
\frac{\partial \log L}{\partial \theta}=\frac{2 n}{\theta}-\frac{n \alpha}{\alpha \theta+1}-n \bar{x}=0 \\
\frac{\partial \log L}{\partial \alpha}=-\frac{n \theta}{\alpha \theta+1}+\sum_{x=1}^{k} \frac{f_{x}}{\alpha+x}=0
\end{gathered}
$$

It can be easily seen that equation (8.2.3) gives $\bar{x}=\frac{\alpha \theta+2}{\theta(\alpha \theta+1)}=\mu_{1}^{\prime}$, mean of TPLD. The equations (8.2.3) and (8.2.4) do not seem to be solved directly. However, Fisher's scoring method can be applied to solve these equations iteratively. We have

$$
\begin{aligned}
& \frac{\partial^{2} \log L}{\partial \theta^{2}}=-\frac{2 n}{\theta^{2}}+\frac{n \alpha^{2}}{(\alpha \theta+1)^{2}} \\
& \frac{\partial^{2} \log L}{\partial \theta \partial \alpha}=-\frac{n}{(\alpha \theta+1)^{2}} \\
& \frac{\partial^{2} \log L}{\partial \alpha^{2}}=\frac{n \theta^{2}}{(\alpha \theta+1)^{2}}-\sum_{x=1}^{k} \frac{f_{x}}{(\alpha+x)^{2}}
\end{aligned}
$$

The maximum likelihood estimates $\hat{\theta}$ and $\hat{\alpha}$ of parameters $\theta$ and $\alpha$ are the solution of the following equations

$$
\left[\begin{array}{cc}
\frac{\partial^{2} \log L}{\partial \theta^{2}} & \frac{\partial^{2} \log L}{\partial \theta \partial \alpha} \\
\frac{\partial^{2} \log L}{\partial \theta \partial \alpha} & \frac{\partial^{2} \log L}{\partial \alpha^{2}}
\end{array}\right]_{\hat{\theta}=\theta_{0}}\left[\begin{array}{c}
\hat{\theta}-\theta_{0} \\
\hat{\alpha}-\alpha_{0}
\end{array}\right]=\left[\begin{array}{c}
\frac{\partial \log L}{\partial \theta} \\
\hat{\alpha}=\alpha_{0}
\end{array}\right] \begin{array}{r}
\frac{\partial \log L}{\partial \alpha} \\
\hat{\theta}=\theta_{0} \\
\hat{\alpha}=\alpha_{0}
\end{array}
$$

where $\theta_{0}$ and $\alpha_{0}$ are initial values of $\theta$ and $\alpha$ as given by the method of moments. These equations are solved iteratively till sufficiently close estimates of $\hat{\theta}$ and $\hat{\alpha}$ are obtained.

\section{Applications of two-parameter lindley distribution}

The two-parameter Lindley distribution (TPLD) has been fitted to a number of lifetime data- sets. In this section, we present the fitting of two-parameter Lindley distribution to five real lifetime data-sets and compare its goodness of fit with the one parameter exponential and Lindley distributions data sets (1-5). 
In order to compare distributions, $-2 \ln L$, AIC (Akaike Information Criterion), AICC (Akaike Information Criterion Corrected), BIC (Bayesian Information Criterion), K-S Statistics (KolmogorovSmirnov Statistics) for five real data - sets have been computed (Table 1). The formulae for computing AIC, AICC, BIC, and K-S Statistics are as follows:

$$
A I C=-2 \ln L+2 k, A I C C=A I C+\frac{2 k(k+1)}{(n-k-1)},
$$

$$
B I C=-2 \ln L+k \ln n
$$

$D=\operatorname{Sup}_{x}\left|F_{n}(x)-F_{0}(x)\right|$, where $k=$ the number of parameters, $\quad=$ the sample size and $F_{n}(x)$ is the empirical distribution function.

The best distribution corresponds to lower $-2 \ln L$, AIC, AICC, BIC, and K-S statistics.

Data set I:This data set represents the lifetime's data relating to relief times (in minutes) of 20 patients receiving an analgesic and reported by Gross et al., ${ }^{23}$

\begin{tabular}{llllllllllll}
\hline 1.1 & 1.4 & 1.3 & 1.7 & 1.9 & 1.8 & 1.6 & 2.2 & 1.7 & 2.7 & 4.1 & 1.8 \\
1.5 & 1.2 & 1.4 & 3 & 1.7 & 2.3 & 1.6 & 2 & & & &
\end{tabular}

Data Set 2: This data set is the strength data of glass of the aircraft window reported by Fuller et al.. ${ }^{24}$

\begin{tabular}{llllllllllll}
\hline 18.83 & 20.8 & 21.657 & 23.03 & 23.23 & 24.05 & 24.321 & 25.5 & 25.52 & 25.8 & 26.69 & 26.77 \\
26.78 & 27.05 & 27.67 & 29.9 & 31.11 & 33.2 & 33.73 & 33.76 & 33.89 & 34.76 & 35.75 & 35.91 \\
36.98 & 37.08 & 37.09 & 39.58 & 44.045 & 45.29 & 45.381 & & & & & \\
\hline
\end{tabular}

Data set 3: This data set represents the waiting times (in minutes) before service of 100 Bank customers and examined and analyzed by Ghitany et al., ${ }^{3}$ for

\begin{tabular}{|c|c|c|c|c|c|c|c|c|c|c|c|}
\hline 0.8 & 0.8 & 1.3 & 1.5 & 1.8 & 1.9 & 1.9 & 2.1 & 2.6 & 2.7 & 2.9 & 3.1 \\
\hline 3.2 & 3.3 & 3.5 & 3.6 & 4 & 4.1 & 4.2 & 4.2 & 4.3 & 4.3 & 4.4 & 4.4 \\
\hline 4.6 & 4.7 & 4.7 & 4.8 & 4.9 & 4.9 & 5 & 5.3 & 5.5 & 5.7 & 5.7 & 6.1 \\
\hline 6.2 & 6.2 & 6.2 & 6.3 & 6.7 & 6.9 & 7.1 & 7.1 & 7.1 & 7.1 & 7.4 & 7.6 \\
\hline 7.7 & 8 & 8.2 & 8.6 & 8.6 & 8.6 & 8.8 & 8.8 & 8.9 & 8.9 & 9.5 & 9.6 \\
\hline 9.7 & 9.8 & 10.7 & 10.9 & 11 & 11 & 11.1 & 11.2 & 11.2 & 11.5 & 11.9 & 12.4 \\
\hline 12.5 & 12.9 & 13 & 13.1 & 13.3 & 13.6 & 13.7 & 13.9 & 14.1 & 15.4 & 15.4 & 17.3 \\
\hline 17.3 & 18.1 & 18.2 & 18.4 & 18.9 & 19 & 19.9 & 20.6 & 21.3 & 21.4 & 21.9 & 23 \\
\hline 27 & 31.6 & 33.1 & 38.5 & & & & & & & & \\
\hline
\end{tabular}
fitting the Lindley² distribution.

Data Set 4: The data set represents the strength of $1.5 \mathrm{~cm}$ glass fibers measured at the National Physical Laboratory, England. Unfortunately, the units of

\begin{tabular}{|c|c|c|c|c|c|c|c|c|c|c|c|}
\hline 0.55 & 0.93 & 1.25 & 1.36 & 1.49 & 1.52 & 1.58 & 1.61 & 1.64 & 1.68 & 1.73 & 1.81 \\
\hline 2 & 0.74 & 1.04 & 1.27 & 1.39 & 1.49 & 1.53 & 1.59 & 1.61 & 1.66 & 1.68 & 1.76 \\
\hline 1.82 & 2.01 & 0.77 & I.II & 1.28 & 1.42 & 1.5 & 1.54 & 1.6 & 1.62 & 1.66 & 1.69 \\
\hline 1.76 & 1.84 & 2.24 & 0.81 & 1.13 & 1.29 & 1.48 & 1.5 & 1.55 & 1.61 & 1.62 & 1.66 \\
\hline 1.7 & 1.77 & 1.84 & 0.84 & 1.24 & 1.3 & $\mathrm{I} .48$ & 1.51 & 1.55 & 1.61 & 1.63 & 1.67 \\
\hline 1.7 & 1.78 & 1.89 & & & & & & & & & \\
\hline
\end{tabular}
measurements are not given in the paper, and they are taken from Smith \& Naylor ${ }^{25}$ 
Data Set 5: The data set is from Lawless. ${ }^{26}$ The data given arose in tests on endurance of deep groove ball bearings. The data are the number of million revolutions before failure for each of the 23 ball bearings in the life tests and they are:

\begin{tabular}{lllllllllllllll}
\hline 17.88 & 28.92 & 33 & 41.52 & 42.12 & 45.6 & 48.8 & 51.84 & 51.96 & 54.12 & 55.56 & 67.8 & & & \\
68.44 & 68.64 & 68.88 & 84.12 & 93.12 & 98.64 & 105.12 & 105.84 & 127.92 & 128.04 & 173.4 &
\end{tabular}

Table I MLE's, - 2In L,AIC,AICC, BIC, K-S statistics of the fitted distributions of data sets I-5

\begin{tabular}{|c|c|c|c|c|c|c|c|c|}
\hline & \multirow{2}{*}{ Model } & \multicolumn{2}{|c|}{ Estimate of Parameters } & \multirow{2}{*}{$-2 \ln L$} & \multirow{2}{*}{ AIC } & \multirow{2}{*}{ AICC } & \multirow{2}{*}{ BIC } & \multirow{2}{*}{ K-S Statistics } \\
\hline & & $\hat{\theta}$ & $\hat{\alpha}$ & & & & & \\
\hline \multirow[t]{3}{*}{ Data I } & Lindley & 0.816118 & & 60.50 & 62.50 & 62.72 & 63.49 & 0.341 \\
\hline & Exponential & 0.526316 & & 65.67 & 67.67 & 67.90 & 68.67 & 0.389 \\
\hline & TPLD & 1.545110 & -0.31285 & 40.71 & 44.7I & $45.4 I$ & 46.70 & 0.204 \\
\hline \multirow[t]{3}{*}{ Data 2} & Lindley & 0.062988 & & 253.99 & 255.99 & 256.13 & 257.42 & 0.333 \\
\hline & Exponential & 0.032455 & & 274.53 & 276.53 & 276.67 & 277.96 & 0.426 \\
\hline & TPLD & 0.103985 & -5.25330 & 231.82 & 235.82 & 236.25 & 238.69 & 0.298 \\
\hline \multirow[t]{3}{*}{ Data 3} & Lindley & 0.186571 & & 638.07 & 640.07 & 640.12 & 642.68 & 0.058 \\
\hline & Exponential & 0.101245 & & 658.04 & 660.04 & 660.08 & 662.65 & 0.163 \\
\hline & TPLD & 0.196210 & 0.337078 & 635.75 & 639.75 & 639.87 & 639.75 & 0.040 \\
\hline \multirow[t]{3}{*}{ Data 4} & Lindley & 0.996116 & & 162.56 & 164.56 & 164.62 & 166.70 & 0.371 \\
\hline & Exponential & 0.663647 & & 177.66 & 179.66 & 179.73 & 181.80 & 0.402 \\
\hline & TPLD & 2.146474 & 0.257373 & 91.56 & 95.56 & 95.63 & 97.36 & $0.36 \mathrm{I}$ \\
\hline \multirow[t]{3}{*}{ Data 5} & Lindley & 0.027321 & & 231.47 & 233.47 & 233.66 & 234.61 & 0.149 \\
\hline & Exponential & 0.013845 & & 242.87 & 244.87 & 245.06 & 246.01 & 0.263 \\
\hline & TPLD & 0.035434 & 10.12355 & 223.52 & 227.52 & 228.12 & 229.79 & 0.098 \\
\hline
\end{tabular}

\section{Conclusion}

In the present paper some of the important mathematical properties including moment generating function, mean deviations, order statistics, Bonferroni and Lorenz curves, entropy and stress strength reliability of two-parameter Lindley distribution (TPLD) of Shanker $\&$ Mishra $^{1}$ have been derived and discussed. The distribution has been fitted to some real lifetime data-sets to test its goodness of fit over exponential and Lindley distributions. It is obvious from the fitting of TPLD that it gives better fitting than exponential and Lindley distributions and hence TPLD is preferable over exponential and Lindley distributions for modeling lifetime data-sets from different fields of knowledge.

\section{Acknowledgement}

None.

\section{Conflict of interest}

The authors declare that they have no financial or non-financial competing interests.

\section{References}

1. Shanker R, Mishra A. A two-parameter Lindley distribution. Statistics in Transition-new series. 2013A;14(1): 45-56.
2. Ghitany ME, Atieh B, Nadarajah S. Lidley distribution and its Application. Mathematics Computing and Simulation. 2008;78(4):493-506.

3. Zakerzadeh H, Dolati A. Generalized Lindley distribution. Journal of Mathematical extension. 2009;3(2):13-25.

4. Nadarajah S, Bakouch HS, Tahmasbi R. A generalized Lindley distribution. Sankhya Series. 2011;73(2):331-359.

5. Deniz E, Ojeda E. The discrete Lindley distribution-Properties and Applications. Journal of Statistical Computation and Simulation. 2011;81(11):1405-1416.

6. Bakouch HS, Al Zaharani B, Al Shomrani A, et al. An extended Lindley distribution. Journal of the Korean Statistical Society. 2012;41(1):75-85.

7. Shanker R, Mishra A. A quasi Lindley distribution. African Journal of Mathematics and Computer Science Research. 2013B;6(4):64-71.

8. Shanker R, Sharma S, Shanker R. A two-parameter Lindley distribution for modelling waiting and survival times data. Applied Mathematics. 2013;4(2):363-368.

9. Elbatal I, Merovi F, Elgarhy M. A new generalized Lindley distribution. Mathematical theory and Modelling. 2013;3(13):30-47.

10. Ghitany M, Al Mutairi D, Balakrishnan N, et al. Power Lindley distribution and associated inference. Computational Statistics and Data Analysis. 2013;64:20-33.

11. Merovci F. Transmuted Lindley distribution. International Journal of Open Problems in Computer Science and Mathematics. 2013;6(2):63-72. 
12. Liyanage GW, Pararai M. A generalized Power Lindley distribution with applications. Asian journal of Mathematics and Applications. 2014;1-23.

13. Ashour S, Eltehiwy M. Exponentiated Power Lindley distribution Journal of Advanced Research. 2014;6(6):895-905.

14. Oluyede BO, Yang T. A new class of generalized Lindley distribution with applications. Journal of Statistical Computation and Simulation. 2014;85(10):2072-2100.

15. Singh SK, Singh U, Sharma VK. The Truncated Lindley distributioninference and Application. Journal of Statistics Applications \& Probability. 2014;3(2):219-228.

16. Sharma V, Singh S, Singh U. The inverse Lindley distribution-A stressstrength reliability model with applications to head and neck cancer data. Journal of Industrial \& Production Engineering. 2015;32(3):162-173.

17. Shanker R, Hagos F, Sujatha S. On modeling of Lifetimes data using exponential and Lindley distributions. Biometrics \& Biostatistics International Journal. 2015;2(5):1-9.

18. Alkarni S.Extended Power Lindley distribution-A new Statistical model for non-monotone survival data. European journal of statistics and probability. 2015;3(3):19-34.

19. Pararai M, Liyanage GW, Oluyede BO. A new class of generalized Power Lindley distribution with applications to lifetime data. Theoretical Mathematics \&Applications. 2015;5(1):53-96.
20. Bonferroni CE. Elementi di Statistca generale. Seeber, Firenze, Itlay. 1930.

21. Renyi A. On measures of entropy and information in proceedings of the $4^{\text {th }}$ Berkeley symposium on Mathematical Statistics and Probability. University of California press, Berkeley, USA, 1961;1:547-561.

22. Gross AJ, Clark VA. Survival Distributions Reliability Applications in the Biometrical Sciences. New York, USA. John Wiley. 1975.

23. Fuller EJ, Frieman S, Quinn J, et al. Fracture mechanics approach to the design of glass aircraft windows: A case study. SPIE Proc. 1994; 2286:419-430.

24. Lindley DV. Fiducial distributions and Bayes' theorem. Journal of the Royal Statistical Society. Series B. 1958;20(1):102-107.

25. Smith RL, Naylor JC. A comparison of Maximum likelihood and Bayesian estimators for the three parameter Weibull distribution. Applied Statistics. 1987;36(3):358-369.

26. Lawless JF. Statistical models and methods for lifetime data. New York, USA; John Wiley and Sons. 1982. 